\title{
Caracterização da assistência odontológica no Piauí: Avaliação do serviço de referência
}

\author{
Characterization of dental care in Piauí: Evaluation of the reference service \\ Caracterización de la atención odontológica en Piauí: Evaluación del servicio de referencia
}

Recebido: 21/05/2021 | Revisado: 29/05/2021 | Aceito: 03/06/2021 | Publicado: 18/06/2021

Sarah de Araújo Mendes Cardoso
ORCID: https://orcid.org/0000-0003-0944-8154
Centro Universitário Santo Agostinho, Brasil
E-mail: sarahamcardoso@gmail.com
Mirna Raquel Rocha de Sousa
ORCID: https://orcid.org/0000-0002-6792-553X
Centro Universitário Santo Agostinho, Brasil
E-mail: mirnaraquel123@hotmail.com
Maria do Amparo Veloso Magalhães
ORCID: https://orcid.org/0000 0002 8330 3247
Centro Universitário Santo Agostinho, Brasil
E-mail: velosocirurgia@yahoo.com.br

\begin{abstract}
Resumo
A qualidade técnica de um serviço pode ser avaliada de forma segura e eficaz, embasada cientificamente. Esse estudo possibilita ao usuário do sistema de saúde público e ao profissional mensurarem o nível de utilidade da assistência que é prestada. Logo, avaliar o serviço odontológico em ambiente de referência viabiliza melhoras significativas para o paciente e profissional, podendo guiar a gestão dos serviços às verdadeiras necessidades da população. Desta forma, o trabalho qualitativo e quantitativo utilizou o setor odontológico do Hospital da Polícia Militar (HPM) de Teresina-PI, para coletar dados que viabilizaram a caracterização dos serviços oferecidos na assistência odontológica, bem como são feitos os registros destes serviços frente a comunidade em que se insere. A pesquisa foi guiada por análise de prontuários dos pacientes, entrevista com formulários estruturados e orientação de cirurgiã-dentista. Na amostra dos prontuários analisados, 64\% não possuíam queixa principal ou características que auxiliassem no diagnóstico e tratamento do paciente, além disso, $42 \%$ dos profissionais confessaram não assinar o documento. O trabalho viabilizou ampliação desse estudo, comparação das percepções de saúde entre paciente e dentista, e possíveis intervenções para melhoria do atendimento para a população.
\end{abstract}

Palavras-chave: Avaliação em saúde; Serviços de saúde bucal; Odontologia em saúde pública.

\begin{abstract}
The technical quality of a service can be assessed safely and effectively, scientifically based. This study allows the user of the public health system and the professional to measure the level of usefulness of the assistance that is provided. Therefore, evaluating the dental service in a reference environment enables significant improvements for the patient and professional, being able to guide the management of services to the real needs of the population. Thus, the qualitative and quantitative work used the dental sector of the Military Police Hospital (HPM) of Teresina-PI, to collect data that enabled the characterization of the services offered in dental care, as well as records of these services in front of the community. in which it is inserted. The research was guided by analysis of patients' medical records, interviews with structured forms and guidance from a dental surgeon. In the sample of analyzed records, $64 \%$ had no main complaint or characteristics that would help in the diagnosis and treatment of the patient, in addition, $42 \%$ of the professionals confessed that they did not sign the document. The work made it possible to expand this study, compare health perceptions between patient and dentist, and possible interventions to improve care for the population.
\end{abstract}

Keywords: Health evaluation; Dental health services; Public health dentistry.

\section{Resumen}

La calidad técnica de un servicio se puede evaluar de forma segura y eficaz, con base científica. Este estudio permite al usuario del sistema público de salud y al profesional medir el nivel de utilidad de la asistencia que se brinda. Por tanto, evaluar el servicio odontológico en un entorno de referencia permite mejoras significativas para el paciente y el profesional, pudiendo orientar la gestión de los servicios a las necesidades reales de la población. Así, el trabajo cualitativo y cuantitativo utilizó el sector odontológico del Hospital de la Policía Militar (HPM) en Teresina-PI, para recolectar datos que permitieron la caracterización de los servicios ofrecidos en la atención odontológica, así como los registros de estos servicios frente a la comunidad en la que se inserta. La investigación se guió por el análisis de los 
registros médicos de los pacientes, entrevistas con formularios estructurados y orientación de un cirujano dental. En la muestra de expedientes analizados, el $64 \%$ no presentaba queja principal ni características que ayudarían en el diagnóstico y tratamiento del paciente, además, el $42 \%$ de los profesionales confesó no firmar el documento. El trabajo permitió ampliar este estudio, comparar las percepciones de salud entre paciente y odontólogo y posibles intervenciones para mejorar la atención a la población.

Palabras clave: Evaluación en salud; Servicios de salud bucal; Odontología en salud pública.

\section{Introdução}

O conceito de qualidade de vida está relacionado ao de autopercepção que, em saúde, pode ser entendida como a interpretação das experiências e do estado de saúde no contexto da vida diária (Vale, Mendes \& Moreira 2013). Por isso, muitos instrumentos de pesquisa são desenvolvidos para avaliarem os níveis de percepções de saúde bucal na população. Logo, a avaliação dos serviços de saúde tem sido uma necessidade amplamente discutida pelas políticas de estado mundiais, considerada um dos caminhos de gestão para buscar a qualidade dos serviços prestados (Bordin, Fadel, Moimaz, Garbin \& Saliba 2017).

A qualidade técnica de um serviço envolve o conhecimento dos profissionais (professores e acadêmicos), o padrão dos equipamentos e a velocidade com que são executados, além da qualidade funcional, bem como a atmosfera do ambiente (biossegurança) (Pêgo et al., 2016). Essas perspectivas foram analisadas por formulários estruturados, para usuários e profissionais, observações em campo e comparações com o padrão estabelecido pelo Sistema Único de Saúde e pela Política Nacional de Saúde Bucal (PNSB), Brasil Sorridente (Brasil - Diretrizes PNSB, 2004).

Segundo estudo de Carreiro, Oliveira, Coutinho, Martins e Haikal (2018), contextos de desigualdade social e menor relação dentista habitante apresentaram-se associados à maior insatisfação com os serviços odontológicos, o que merece destaque. Já no que tange às variáveis individuais, verificou-se maior chance de insatisfação entre os que se autodeclararam amarelos/negros/ pardos/indígenas, os com menor escolaridade, os que se consultaram por problemas bucais, os que estavam insatisfeitos com os dentes e boca e os que apresentaram impacto bucal no desempenho diário. A insatisfação com os serviços odontológicos apresentou distribuição que variou com o nível de desenvolvimento das regiões brasileiras, com menores proporções de insatisfação registradas nas regiões mais desenvolvidas: 10,0\% no Sul e 10,2\% no Centro-Oeste. Inversamente, as maiores proporções de insatisfação foram identificadas nas regiões menos desenvolvidas: $15,3 \%$ no Norte e $14,1 \%$ no Nordeste (Carreiro et al., 2017).

Iniciativas de reorganização dos processos de trabalho, assim como otimização dos fluxos internos podem contribuir demasiadamente para a melhoria da qualidade dos serviços e consequentemente na satisfação dos usuários, devendo, dessa forma, a organização dos serviços e das práticas de saúde ser capaz de realizar uma apreensão ampliada das necessidades da população sob a sua responsabilidade (Brandão, Giovanella \& Campos, 2013). Ressalta-se que o processo da avaliação de serviços odontológicos na perspectiva dos usuários e as variáveis pesquisadas é mutável, portanto, causas e efeitos certamente variam ao longo da prestação de serviço que é dada e as distintas políticas envolvidas (Carreiro et al., 2017).

As pesquisas que avaliam os serviços de saúde podem promover, direta ou indiretamente, impacto positivo na condição de saúde da população, pois a análise direciona o entendimento de como estão sendo efetuados os serviços e como podem melhorar (Carreiro et al., 2017, Carreiro et al., 2018). Nessa perspectiva, escolheu-se o Hospital da Polícia Militar da cidade de Teresina, capital do estado do Piaú, sendo um serviço de referência e que oferece atendimento nas três esferas de atenção à saúde, para a caracterização da assistência odontológica prestada à população.

Por essas necessidades de avaliar e comparar a visão de usuários e de profissionais acerca da satisfação dos aspectos inerentes ao serviço odontológico tem-se o fundamento e a motivação do presente artigo. No âmbito populacional, há possibilidades variadas quanto às análises feitas dos serviços de saúde, nisso o presente artigo, avaliou se o setor odontológico do hospital de referência presta serviço satisfatório à população. Procurou-se caracterizar esse serviço para usuários e dentistas que compõem a equipe hospitalar. É esperado que as ações desenvolvidas no serviço estejam de acordo com o padrão 
estabelecido pela legislação atual no que diz respeito ao atendimento público em odontologia.

\section{Metodologia}

O presente trabalho trata-se de um estudo qualitativo e quantitativo, que visa à pesquisa de campo e análise de dados. A pesquisa de campo decorreu por formulários estruturados, adaptados dos protocolos do Programa Nacional de Avaliação dos Serviços de Saúde (PNASS) (Brasil, 2015), que é o instrumento de base nacional proposto pelo Ministério da Saúde para qualificar o serviço prestado. Os dados foram coletados por meio de questionários, entrevistas, grupos focais, reuniões e observações com a participação do pesquisador (Macêdo \& Evangerlandy, 2018). O questionário obteve informações tanto dos pacientes e profissionais, quanto possibilitou conhecer como funciona o ambiente em questão (Brasil, 2015). O estudo qualitativo tratou-se de critérios subjetivos aos objetivos que buscam compreender os fenômenos sociais (educação, sociologia, psicologia, antropologia, dentre inúmeras outras áreas do conhecimento).

O campo de pesquisa foi o Hospital Dirceu Arcoverde, também denominado como Hospital da Polícia Militar do Piauí (HPMPI). Um Hospital especializado no atendimento à Assistência Médico-Hospitalar, tendo como principal finalidade prevenir doenças, promover a saúde, estimular a educação e desenvolver pesquisa. Dispõe de um quadro de profissionais responsáveis e comprometidos que estão trabalhando para o bem-estar da população Militar e Civil com a Filosofia - "Humanizando e Cuidando Bem de Sua Saúde”, com uma gestão moderna pautada nos princípios de Eficiência e Eficácia.

A amostra constou de 14 cirurgiões-dentistas, civis e militares, de diversas especialidades, que trabalham na assistência em odontologia no HPMPI; 26 pacientes, correspondente a média de atendimentos semanais e consulta de 36 prontuários selecionados aleatoriamente nos arquivos do serviço. Foram incluídos os cirurgiões dentistas do serviço disponíveis, os pacientes atendidos no período de uma semana, sem distinção de gênero, e consultados 36 prontuários dos últimos 5 anos, selecionados ao acaso. Foram excluídos os profissionais e pacientes que se recusaram a fazer parte do estudo, que se recusaram a assinar o Termo de Consentimento Livre e Esclarecido e os menores de 18 anos (Appolinário, 2006). Foi aplicado um questionário por meio de entrevista individualizada, com os cirurgiões dentistas, outro com os pacientes, conduzido por orientador treinado para angariar as informações necessárias e acolher dúvidas, sem influenciar nas respostas, essa etapa ocorreu em ambiente reservado. Um questionário foi preenchido com as indagações sobre os registros dos prontuários.

A pesquisa foi realizada mediante autorização por escrito do representante legal da Instituição supracitada, em consonância com a Portaria do Ministério da Saúde, Conselho Nacional de Ética em Pesquisa, que regulamenta as questões operacionais e éticas dos trabalhos científicos envolvendo seres humanos. O presente estudo foi submetido a análise pelo Comitê

de Ética em Pesquisa do Centro Universitário Santo Agostinho (CEP/UNIFSA), com o que determina as Resoluções nº 466/2012 e 510/2016 do Conselho Nacional Saúde do Ministério da Saúde (CNS/MS). Os dados foram analisados através de comparações com estudos preexistentes acerca do tema e apresentados em tabelas.

\section{Resultados e Discussão}

Os primeiros dados coletados resultaram da análise dos prontuários (Tabela 1), dos quais $64 \%$ estavam com as informações incompletas sobre o paciente e $83 \%$ não estavam preenchidos o odontograma, o pré e pós tratamento. Na amostra analisada, os $64 \%$ não possuíam exposição de queixa principal ou características que auxiliem no diagnóstico e tratamento do paciente. Há dados coletados sobre a participação legal e consciente do paciente em concordar com o plano de tratamento proposto, com $97 \%$ dos prontuários não assinados pelo paciente. Além da ausência da assinatura do paciente, $42 \%$ da amostra analisada não apresentava assinatura do profissional que realizou o atendimento. 
Tabela 1: Informações do prontuário odontológico coletadas através de questionário estruturado. N=36.

\begin{tabular}{|l|l|l|}
\hline Questionário do Prontuário & SIM & NÃO \\
\hline $\begin{array}{l}\text { O prontuário possui as informações } \\
\text { completas do paciente? }\end{array}$ & $36 \%$ & $64 \%$ \\
\hline $\begin{array}{l}\text { No prontuário possui as condições } \\
\text { dentárias do pré e pós tratamento? }\end{array}$ & $17 \%$ & $83 \%$ \\
\hline $\begin{array}{l}\text { No prontuário há o consentimento } \\
\text { do paciente para realizar o plano de } \\
\text { tratamento? }\end{array}$ & $3 \%$ & $97 \%$ \\
\hline $\begin{array}{l}\text { O profissional assina o prontuário } \\
\text { sendo responsável? }\end{array}$ & $58 \%$ & $42 \%$ \\
\hline
\end{tabular}

Fonte: Autores (2020).

Há a necessidade de dados como queixa principal, história da doença atual, história médica e odontológica, familiar e social serem devidamente coletados, assim como uso de medicamentos, alergias, alterações sistêmicas e patologias diversas são pontos importantes para diagnóstico e planejamento do atendimento (Silva, do Prado, Rodrigues, Picoli \& Franco 2016). No prontuário odontológico deve ser encontrado o diagnóstico, prognóstico, eventuais intercorrências, ficha clínica, radiografia e o acompanhamento da evolução do paciente (Almeida, Delwing, Assis, Furtado \& Azevedo 2017). O autor supracitado confirma também que esse documento tem a função de comprovar o zelo profissional com a saúde do paciente e se estabelece como veículo de comunicação entre profissional e paciente.

É possível compreender que sem o exame físico não há como estabelecer o grau de saúde que o paciente se apresentava no momento do atendimento e que registrar taxas sistêmicas e características físicas do paciente compõe um atendimento de qualidade. Portanto, sua finalidade clínica também pode ensejar efeitos jurídicos, sendo, pois, necessário uma elaboração que cumpra os requisitos administrativos, éticos e legais, visto que a falha em um desses quesitos pode invalidar o prontuário (Almeida et al., 2017).

Da perspectiva legal, o cirurgião-dentista é visto como um fornecedor de produtos e serviços e o paciente é o consumidor, em uma relação de consumo de acordo com o Código de Defesa do Consumidor (CDC) (CFO, 2012), em vigor desde 1990. Assim a assinatura do paciente no prontuário é a forma de demonstrar a relação das partes, bilateralmente, constituindo importante comprovação de ciência de ambos (Silva et al., 2016). Esse ponto foi constatado de forma parcialmente satisfatória nos prontuários odontológicos, onde 58\% dos prontuários da amostra (Tabela 1) estavam assinados pelo dentista, comprovando sua participação na construção do mesmo e na responsabilização do diagnóstico e decisão de tratamento. Porém, 97\% da amostra revelou que o paciente não assina o prontuário, já que sua participação é validada quando adere ao plano do hospital, podendo perder de forma parcial sua participação legal na contratualidade do serviço, pois sem sua assinatura a relação bilateral do documento perde valor, característica que desqualifica o prontuário utilizado. Silva et al. (2016), afirma que o paciente deve assinar o prontuário e todos os documentos referidos a ele (anamnese, plano de tratamento, TCLE) no início e no fim do seu tratamento e o profissional deve compreender que as assinaturas fundamentam o conteúdo e validam o documento. Esses aspectos podem ser verificados na Tabela 1 que possui as indagações quanto às participações do cirurgião-dentista e do paciente como responsáveis no prontuário.

De acordo com Silva et al. (2007), o registro correto do aspecto bucal possibilita ao dentista contribuir com a justiça nos casos de identificação humana e faz desse documento elemento de prova, sendo obrigatória sua elaboração e manutenção de forma legível e atualizada, referindo-se às informações das condições dentárias do pré e pós-operatório. Do total de prontuários analisados, 83\% (Tabela 1) não possuem a caracterização requerida das condições dentárias do paciente. Representando um documento não utilizado de forma judicial ou em casos de identificação humana e elemento de prova.

De acordo com o inciso VIII do artigo $5^{\circ}$ do Código de Ética Odontológica (2012), "é dever fundamental dos profissionais da Odontologia elaborar e manter atualizados os prontuários dos pacientes, conservando-os em arquivo próprio". 
A exigência ética da devida elaboração e atualização do prontuário odontológico é necessária para resguardar profissional e paciente de possíveis litígios que os envolva, seja por acusação de erro profissional, iatrogenia, cobrança de honorários, ou qualquer motivo que leve à desarmonia das partes (Ramos, 2005; Almeida et al., 2017).

\subsection{Análise do questionário aplicado aos cirurgiões-dentistas}

A Tabela 2 apresenta os dados do questionário de satisfação do cirurgião-dentista, quanto aos instrumentais e material odontológico ofertado no serviço, onde $57 \%$ dos profissionais afirmaram satisfação quanto aos instrumentais oferecidos para os atendimentos e 57\% também afirmaram suficiência na quantidade de material odontológico. Outro dado de análise do questionário aplicado ao cirurgião-dentista, é quanto ao ambiente de trabalho e suas expectativas, revelando que $72 \%$ dos profissionais sentem suas expectativas supridas dentro do serviço. E por último, o dado do questionário quanto ao preenchimento do prontuário, sendo que $42 \%$ dos profissionais confessaram não assinar e/ou assinar as vezes o documento.

Tabela 2: Informações da satisfação do Cirurgião-Dentista coletados através de questionário estruturado. N=14

\begin{tabular}{|l|l|l|l|}
\hline $\begin{array}{l}\text { Questionário do } \\
\text { Cirurgião-Dentista }\end{array}$ & SIM & Ñ̃O & ÅS VEZES \\
\hline $\begin{array}{l}\text { A quantidade de } \\
\text { instrumentais oferecidos } \\
\text { no serviço supre as } \\
\text { necessidades? }\end{array}$ & $57 \%$ & $43 \%$ & 0 \\
\hline $\begin{array}{l}\text { A quantidade de material } \\
\text { odontológico é suficiente } \\
\text { para demanda? }\end{array}$ & $57 \%$ & $43 \%$ & 0 \\
\hline $\begin{array}{l}\text { O ambiente de trabalho } \\
\text { supre as expectativas? }\end{array}$ & $72 \%$ & $14 \%$ & $14 \%$ \\
\hline $\begin{array}{l}\text { Você preenche o } \\
\text { formulário odontológico? }\end{array}$ & $57 \%$ & $22 \%$ & $21 \%$ \\
\hline
\end{tabular}

Fonte: Autores (2020).

A necessidade de se compreender a qualidade dos serviços prestados por profissionais da saúde é alvo de discussões e avaliações que norteiam mudanças nas gestões do atendimento odontológico (Bordin et al., 2017). Essas avaliações devem ser verificadas tanto da perspectiva do paciente quanto do profissional, sendo que trabalhadores da saúde atribuem maior importância às competências técnicas do atendimento. Na aplicação do questionário para o cirurgião-dentista pode-se observar aspectos relacionados ao instrumental e o material disponível para o uso do profissional.

De acordo com as Diretrizes da Política Nacional de Saúde Bucal (Brasil, 2004), é preciso que haja o desenvolvimento de políticas de suprimento de instrumentos e material de consumo e de conservação, manutenção e reposição dos equipamentos odontológicos, de modo a garantir condições adequadas de trabalho. Esse aspecto assegura a qualidade do trabalho do cirurgiãodentista, visto que sua atuação é limitada quanto aos materiais e instrumental disponíveis.

É possível estabelecer uma análise sob a perspectiva do grau de satisfação quanto ao ambiente de trabalho e a atuação do profissional, pois para Moimaz et al. (2015), a prática odontológica expõe o dentista a riscos físicos, químicos, ergonômicos, mecânicos ou de acidente, que podem ser causados pela ausência de conforto, higiene ou comunicação entre os profissionais. Por isso o autor afirma ser necessário um ambiente de trabalho adequado com condutas preventivas e boas relações interpessoais para que seja possível que o odontólogo desenvolva todas suas competências, minimizando os desgastes físicos e psicológicos.

Outro fator que prejudica a satisfação no ambiente de trabalho tornando-o debilitante psicológico e mental é o estresse. Sendo a odontologia considerada uma das profissões mais estressantes, os números de fatores de estresse crescem, passando desde problemas financeiros, de gestão e sociais, aos quais nem sempre os dentistas estão preparados. Esses fatores podem afetar de forma significativa a sua saúde física, mental ou ambos (Guanabara \& Martins, 2017). 
Como já discutido na Tabela 1, a importância do prontuário para um pleno atendimento é inegável, seja na perspectiva do desenvolvimento do tratamento como em aspectos legais e contratuais. Segundo Peixoto, Feitoza, de Albuquerque e Nascimento (2019), o cirurgião-dentista torna-se responsável por suas ações ou omissões durante o atendimento no âmbito ético, civil e penal, sendo os componentes do prontuário participantes do diagnóstico, escolha do tratamento, prognóstico e prova da responsabilização do dentista e acordo do paciente.

A taxa de confirmação de preenchimento do prontuário por parte do dentista (Tabela 2) afirma que $57 \%$ observa o prontuário como importante, no entanto essa afirmação é questionada pelos dados da Tabela 1, que evidenciam alto percentual de falha nesse preenchimento, com informações incompletas do paciente e ausência das condições dentárias do pré e pósoperatório. Para Andrade, dos Santos e Canettieri (2018), o conhecimento da importância do prontuário odontológico deve iniciar-se na formação acadêmica dos cirurgiões-dentistas, os quais vivenciam no ambiente de graduação o primeiro contato com a prática da anamnese e os aspectos necessários para um correto diagnóstico e escolha de tratamento viável para cada paciente.

\subsection{Análise do questionário aplicado aos pacientes}

A Tabela 3 apresenta os dados do questionário aplicado ao paciente, quanto à escolaridade, percebendo-se diversidade no nível educacional dos usuários, desde $4 \%$ de pacientes com nível fundamental incompleto, $23 \%$ nível médio completo, $15 \%$ com superior incompleto e $4 \%$ com especialização; principais procedimentos procurados pelo paciente no serviço odontológico, sendo possível destacar um número significante de restaurações (54\%) o que reflete na busca de tratamentos mais preventivos e conservadores, seguidos de endodontia (27\%); a satisfação do paciente quanto ao atendimento do profissional e dos funcionários da recepção, obtendo $81 \%$ de respostas satisfatórias quanto aos dentistas e $38 \%$ caracterizou como muito bom o atendimento dos funcionários da recepção; o nível de confiança durante o atendimento na equipe de profissionais de saúde bucal, sendo possível analisar dado satisfatório sobre a atuação do profissional, com $96 \%$ das respostas colhidas demonstrarem confiança no dentista. E por fim, a satisfação do paciente quanto ao conhecimento do local de queixa do atendimento, sendo que $23 \%$ dos pacientes não sabem onde podem ser ouvidos quando há reclamação a ser feita e $77 \%$ dos usuários do serviço sabem onde podem fazer reclamação dentro do serviço odontológico.

Tabela 3: Informações da satisfação do paciente, coletados através de questionário estruturado. N=26.

\begin{tabular}{|c|c|c|c|c|c|c|c|}
\hline \multicolumn{8}{|c|}{ Grau de escolaridade dos pacientes } \\
\hline $\begin{array}{l}\text { Alfabetizado } \\
-4 \%\end{array}$ & $\begin{array}{l}\text { Nível } \\
\text { fundamental } \\
\text { incompleto } \\
-4 \%\end{array}$ & $\begin{array}{l}\text { Nível } \\
\text { fundamental } \\
\text { completo } \\
-8 \%\end{array}$ & $\begin{array}{l}\text { Nível } \\
\text { médio } \\
\text { incompleto } \\
-15 \%\end{array}$ & $\begin{array}{l}\text { Nível } \\
\text { médio } \\
\text { completo } \\
-23 \%\end{array}$ & \begin{tabular}{|l|} 
Nível \\
superior \\
incompleto \\
$-15 \%$ \\
\end{tabular} & \begin{tabular}{|l|} 
Nível \\
superior \\
completo \\
$-27 \%$ \\
\end{tabular} & Especialização - 4\% \\
\hline \multicolumn{8}{|c|}{ Procedimentos buscados } \\
\hline Exodontia - & & Endodontia - & $27 \%$ & Profilaxia & $-15 \%$ & Restauraç & $0-54 \%$ \\
\hline \multicolumn{8}{|c|}{ Avaliação quanto ao atendimento na recepção } \\
\hline Muito bom - & $38 \%$ & Bom $-31 \%$ & & & Ruim $-27 \%$ & & Não sabe / Não respondeu - 4\% \\
\hline \multicolumn{8}{|c|}{ Avaliação quanto ao atendimento odontológico } \\
\hline Satisfatório- & $81 \%$ & & Regular -1 & & & Não sabe & Não respondeu $-8 \%$ \\
\hline \multicolumn{8}{|c|}{ Segurança/Confiança na equipe de saúde bucal durante o atendimento } \\
\hline Sim $-96 \%$ & & & & Não $-4 \%$ & & & \\
\hline \multicolumn{8}{|c|}{ Sabe onde reclamar quando não é bem atendido? / Ouvidoria } \\
\hline Sim $-77 \%$ & & & & Não -239 & & & \\
\hline
\end{tabular}

Fonte: Autores (2020).

A avaliação de um paciente é responsável por refletir a eficácia de um serviço oferecido. Dessa maneira, a atenção dada a concepção do usuário quanto ao serviço prestado, tem por finalidade prestar um atendimento de qualidade e mais humanitário. Os profissionais que observam a integralidade do indivíduo entendem de maneira mais eficiente quais são as necessidades destes, sendo capaz de analisar as limitações dos serviços através da compreensão do perfil do paciente (Bottan, de Liz, SchlichTelles 
\& Neto Uriarte 2006; Freitas, 2014).

A compreensão das desigualdades no sistema de saúde permite impulsionar mudanças comportamentais e melhorar resultados no acesso, potencializando-o e permitindo o monitoramento e o gerenciamento das ações oferecidas (Fonseca, Fonseca \& Meneghim 2017). Os resultados quanto aos procedimentos buscados (Tabela 3), são semelhantes aos encontrados por Albuquerque et al. (2016), que justifica a maior procura por tratamentos preventivos pela visão dos pacientes de evitar a progressão da doença cárie e prevenir novos casos de dor.

Os dados quanto ao atendimento da recepção e dos cirurgiões-dentistas, demonstram de maneira direta a qualidade do serviço prestado segundo a visão dos usuários, que está mais relacionado com a forma pelo qual ele é tradado do que com a parte técnica. Uma experiência ruim pode gerar consequências desastrosas na vida do paciente e até mesmo aos fornecedores do serviço, por isso a qualidade é de fundamental importância para a fidelização e bem-estar do paciente (Bottan et al., 2006; Coelho et al., 2020). Assim, o conhecimento sobre o nível de satisfação do paciente, assim como a avaliação da confiabilidade, mede se o serviço está sendo capaz de atender as expectativas, nesse contexto a percepção do paciente é necessária para a avaliação da qualidade do serviço (Lima, Cabral \& Vasconcelos, 2010). É de responsabilidade da equipe de saúde bucal criar e desenvolver práticas éticas, comportamentos morais, atualizações científicas constantes para oferecer um atendimento de qualidade e confiança, para isso é necessário um eficiente e organizado sistema de documentação, um relacionamento amistoso e, por vezes, até caritativo com seus pacientes, guiando-se pelas disposições presentes no Código de Ética Odontológico (Enio Junior \& Oliveira, 2017).

Ressalta-se por fim, que o objetivo de integrar e estimular práticas que ampliem o acesso dos usuários ao processo de avaliação das ações e serviços públicos de saúde foi criada em 2003 ouvidoria geral do SUS, o que tem contribuído de maneira positiva para o fortalecimento da participação social e o usuário passa a ser protagonista da gestão de saúde, assim assegurando seus direitos (de Sousa et al, 2017). Segundo de Sousa et al. (2017), é importante ressaltar a garantia de melhora na qualidade do serviço que é ofertado quando se busca conhecer a satisfação, elogios e sugestão dos usuários. A ouvidoria é um órgão cuja função principal é "ouvir" a manifestação acerca de questionamentos em relação ao serviço, intervindo na relação entre o usuário e a administração pública. Além disso, a Ouvidoria quando presente em um órgão administrativo pode ser utilizada como instrumento de gestão, pois suas informações geradas auxiliam desde o planejamento até a formulação de propostas e avaliação de possíveis mudanças e adaptações (Sá \& Fernandez, 2019).

As avaliações realizadas por essas análises pôde caracterizar o atendimento odontológico prestado pelo serviço de referência na capital do Piauí, pois um estudo acerca dos cuidados em saúde bucal devem revelar seu exercício para que se compreenda seu impacto na vida do usuário do Serviço Público no Brasil (Delgado et al., 2021).

\section{Conclusão}

O perfil dos pacientes que buscaram a instituição possuía um nível médio de escolaridade e demonstraram satisfação com a prestação de serviços recebidos, além de demonstrarem um grau elevado de confiança nos cirurgiões-dentistas que trabalham na instituição. Um aspecto relevante quanto aos atendimentos mais buscados verificou-se no alto percentual de procedimentos curativos e restauradores, o que gera reflexão na necessidade de uma maior promoção de saúde bucal nessa população, onde a prevenção possa ganhar espaço compondo o índice da qualidade de vida da comunidade em estudo.

Os profissionais demonstraram estar satisfeitos com a realidade atual do seu ambiente de trabalho, com expectativas de melhorias. O serviço se mostrou muito importante para a população e tem perspectiva de ampliação.

As limitações da pesquisa consistiram na ausência de um sistema organizacional dos prontuários odontológicos, os quais sendo manuais não possuíam controle numérico, além do seu manuseio por vários funcionários em turnos diferentes.

É possível concluir que há uma excelente qualidade de profissionais e serviços prestados com clara satisfação da 
população atendida, porém, necessita de uma mudança urgente na forma de fazer os registros legais dos atendimentos, podendo ser implantado o sistema de prontuário eletrônico, sendo um possível viés a ser avaliado em próximos estudos.

\section{Referências}

Albuquerque, Y. E., Zuanon, Â. C. C., Pansani, C. A., Giro, E. M. A., Lima, F. C. B. D. A., Pinto, L. A. M. D. S., ... \& Brighenti, F. L. (2016). Perfil do atendimento odontológico no Serviço de Urgência para crianças e adolescentes da Faculdade de Odontologia de Araraquara (FOAr)-UNESP. Revista de Odontologia da UNESP, 45(2), 115-120.

Almeida, S. M., Delwing, F., Assis, M. D. P. S., Furtado, F. M. S., \& Azevedo, J. A. P. (2017). Responsabilidade profissional e documentação Odontológicarevisão de literatura. Rev Bahiana Odontol, 8(1), 19-25.

Andrade, A. C. M., dos Santos Zanelato, V., \& Canettieri, A. C. V. (2018). Avaliação da percepção dos cirurgiões-dentistas de São José dos Campos (SP) sobre a importância legal do prontuário odontológico. Revista Brasileira de Odontologia Legal, 5(3), 2-11.

Appolinário, F. (2006). Metodologia da ciência: filosofia e prática da pesquisa. Thomson.

Brandão, A. L. D. R. B. D. S., Giovanella, L., \& Campos, C. E. A. (2013). Avaliação da atenção básica pela perspectiva dos usuários: adaptação do instrumento EUROPEP para grandes centros urbanos brasileiros. Ciência \& Saúde Coletiva, 18, 103-114.

Brasil. (2004). Diretrizes da política nacional de saúde bucal. Ministério da Saúde. Brasília

Brasil. (2015). PNASS: Programa Nacional de Avaliação de Serviços de Saúde 2015. Ministério da Saúde, Secretaria-Executiva, Departamento de Regulação, Avaliação e Controle de Sistemas. Ministério da Saúde, Brasília.

Bordin, D., Fadel, C. B., Moimaz, S. A. S., Garbin, C. A. S., \& Saliba, N. A. (2017). Estudo comparativo da satisfação de usuários e profissionais da saúde com o serviço público odontológico. Ciência \& Saúde Coletiva, 22, 151-160.

Bottan, E. R., de Liz Sperb, R. A., SchlichTelles, P., \& Neto, M. U. (2006). Avaliação de serviços odontológicos: a visão dos pacientes. Revista da ABENO, $6(2), 128-133$.

Carreiro, D. L., Oliveira, R. F. R., Coutinho, W. L. M., Martins, A. M. E. D. B. L., \& Haikal, D. S. A. (2018). Avaliação da satisfação com a assistência odontológica na perspectiva de usuários brasileiros adultos: análise multinível. Ciência \& Saúde Coletiva, 23, 4339-4349. DOI:10.1590/1413812320182312.32792016

Carreiro, D. L., Souza, J. G. S., Coutinho, W. L. M., Ferreira, R. C., Ferreira, E. F., \& Martins, A. M. E. D. B. L. (2017). Uso de serviços odontológicos de forma regular na população de Montes Claros, MG, Brasil. Ciência \& Saúde Coletiva, 22, 4135-4150.

CFO. (2012). Conselho Federal de Odontologia - Código de Ética Odontológico - Rio de Janeiro

Coelho, I. V., de Melo, A. R. F., Caetano, R. M., de Melo Silva, C. L., Habibe, R. C. H., \& Habibe, C. H. (2020). Avaliação da satisfação do paciente atendido na Clínica Integrada Odontológica do UniFOA. Brazilian Journal of Health Review, 3(1), 673-683.

de Sousa Gomes, A. C., do Nascimento Bernardo, G., dos Reis Carvalho, F., da Costa Oliveira, L. D., Lima, T. S., \& Mendes, M. M. (2017). O papel das ouvidorias e dos conselhos de saúde no sus: uma revisão integrativa de literatura. Revista Eletrônica Gestão e Saúde, 8(1), 140-161.

Delgado, R. A. S., Sobral, A. P. T., Gonçalves, M. L. L., Souza, M. R., Fragoso, Y. D., Santos, E. M., \& Mendes, G. D. (2021). Avaliação do impacto do Programa Odontológico Preventivo de Bebês: Coorte retrospectivo. Research, Society and Development, 10(5).

Freitas, L. A. (2014). O marketing de relacionamento como ferramenta estratégica na reputação das organizações de saúde. Revista Mídia \& Contexto-ISSN: $2358-3312,2(1), 1-14$.

Fonseca, E. P. D., Fonseca, S. G. O. D., \& Meneghim, M. D. C. (2017). Análise do acesso aos serviços odontológicos públicos no Brasil. ABCS health sci; 42(2), $85-92$.

Guanabara, D. Q., \& Martins, L. F. B. (2017). Avaliação das condições de trabalho dos cirurgiões-dentistas dos Centros de Especialidades Odontológicas de Baturité e Quixeramobim, Ceará. Mostra Científica do Curso de Odontologia, 2(1).

Junior, E. F., \& de Oliveira Trindade, G. (2017). Responsabilidade do Cirurgião Dentista Frente ao Código de Defesa do Consumidor. Cadernos UniFOA, 5(12), $63-70$

Lima, A. C. S. D., Cabral, E. D., \& Vasconcelos, M. M. V. B. (2010). Satisfação dos usuários assistidos nos Centros de Especialidades Odontológicas do município do Recife, Pernambuco, Brasil. Cadernos de Saúde Pública, 26, 991-1002.

Macêdo, F. C. D. S., \& Evangerlandy, G. M. (2018). Pesquisa: passo a passo para elaboração de trabalhos científicos. Teresina: Macêdo, FCS

Moimaz, S. A. S., Costa, A. C. O., Saliba, N. A., Bordin, D., Rovida, T. A. S., \& Garbin, C. A. S. (2015). Condições de trabalho e qualidade de vida de cirurgiõesdentistas no Sistema Único de Saúde. Revista Ciência Plural, 1(2), 68-78.

Pêgo, S. P. B., Dias, V. O., Nascimento, J. E., Junior, H. M., Martelli, D. R. B., Santos, M. L., \& Sampaio, C. A. (2016). Avaliação dos serviços odontológicos prestados por acadêmicos: percepção do usuário. Revista Intercâmbio, 7, pag-138

Peixoto, F. B., Feitoza, R. R., de Albuquerque Machado, C. T., \& Nascimento, J. D. R. (2019). Responsabilidade do Cirurgião-dentista com o prontuário clínico. Revista Eletrônica Acervo Saúde, (21), e575-e575 
Research, Society and Development, v. 10, n. 7, e16710716360, 2021

(CC BY 4.0) | ISSN 2525-3409 | DOI: http://dx.doi.org/10.33448/rsd-v10i7.16360

Ramos, D. I. D. A. (2005). Prontuário odontológico: aspectos éticos e legais. Dissertação. Faculdade de Odontologia de Piracicaba da Universidade Estadual de Campinas; São Paulo.

Sá, D. A. D., \& Fernandez, M. (2019). Protocolo metodológico para análise das informações gerenciais da área de gestão da informação da ouvidoria-geral do SUS. Ministério da Saúde, Recife, FIOCRUZ.

Silva, R. F., do Prado, M. M., Rodrigues, L. G., Picoli, F. F., \& Franco, A. (2016). Importância ético-legal e significado das assinaturas do paciente no prontuário odontológico. Revista Brasileira de Odontologia Legal, 3(1), 70-83.

Silva, R. F. D., Portilho, C. D. M., Reges, R. V., Leles, C. R., Freitas, G. C. D., \& Daruge Júnior, E. (2007). Importância pericial dos registros odontológicos decorrentes de tratamento restaurador. Rev. dental press estét, 32-38.

Vale, E. B. D., Mendes, A. D. C. G., \& Moreira, R. D. S. (2013). Autopercepção da saúde bucal entre adultos na região Nordeste do Brasil. Revista de Saúde Pública, 47, 98-108. DOI:10.1590/S0034-8910.2013047004893 